INPLASY

PROTOCOL

To cite: Alawaji et al. Accuracy of Estimating Periodontitis and Its Risk Association Using Partial-Mouth Recordings for Surveillance Studies. A Systematic Review and MetaAnalysis. Inplasy protocol 202160032. doi: 10.37766/inplasy2021.6.0032

Received: 10 June 2021

Published: 10 June 2021

Corresponding author: Yasmine Alawaji

alawajiy@student.ubc.ca

Author Affiliation:

The University of British Columbia.

Support: No financial support.

Review Stage at time of this submission: Piloting of the study selection process.

Conflicts of interest:

None declared.

\section{Accuracy of Estimating Periodontitis and Its Risk Association Using Partial-Mouth Recordings for Surveillance Studies. A Systematic Review and Meta-Analysis}

Alawaji, $\mathrm{Y}^{1} ;$ Alshammari, A2; Carvalho, R3; Mostafa, N4; Aleksejuniene, $\mathrm{J}^{5}$.

Review question / Objective: Should we use partial mouth recording (PRP) for assessment of periodontitis in surveillance studies? Specific objectives: to conduct an upto-date systematic review and meta-analysis regarding the assessment of accuracy of using PRP to estimates the prevalence, estimate of extent and severity and risk associations to periodontal disease.

Condition being studied: Accuracy of partial mouth recording protocols compared to full mouth recording protocol in assessment of periodontal disease prevalence, risk associations, estimates of severity and extent.

Information sources: Medline via OVID and PubMed, Embase through OVID, Grey literature at the Networked Digital Library of Theses and Dissertations, Proquest Dissertations and Theses Global, OpenGrey, Biosis, and Grey Matter. References of the individual articles and related (similar) articles.

INPLASY registration number: This protocol was registered with the International Platform of Registered Systematic Review and Meta-Analysis Protocols (INPLASY) on 10 June 2021 and was last updated on 10 June 2021 (registration number INPLASY202160032).

\section{INTRODUCTION}

Review question / Objective: Should we use partial mouth recording (PRP) for assessment of periodontitis in surveillance studies? Specific objectives: to conduct an up-to-date systematic review and metaanalysis regarding the assessment of accuracy of using PRP to estimates the prevalence, estimate of extent and severity and risk associations to periodontal disease.

Rationale: Previous studies that evaluated the accuracy of using PRPs mainly focused on examining the prevalence of 
periodontitis and/or summary estimates of extent and severity. Not until recently, studies attempted to assess the accuracy in estimating the risk association with periodontitis using PRPs. There is no published systematic review that assess the accuracy of studying the periodontitis risk association. Also, previous systematic review that assessed the prevalence and estimates of severity and extent using PRP was published in 2013 and up-to-date evidence is needed.

Condition being studied: Accuracy of partial mouth recording protocols compared to full mouth recording protocol in assessment of periodontal disease prevalence, risk associations, estimates of severity and extent.

\section{METHODS}

Search strategy: Medical Subject heading (Mesh) terms and keywords that will be used include: periodontal attachment loss, periodontitis, prevalence, extent, severity, partial mouth recording, Half mouth, and partial recording protocol. No limits or filters will be used when conducting the search. Databases and sources that will be used include Medline via OVID and PubMed using Mesh terms, key words and related articles. Embase through OVID. Grey literature at the Networked Digital Library of Theses and Dissertations, Proquest Dissertations and Theses Global, OpenGrey, Biosis, and Grey Matter. References of the individual articles will be checked.

Participant or population: Subjects from any age group with permanent dentition.

Intervention: Partial mouth recording (PRP).

Comparator: Full mouth recording (FRP).

Study designs to be included: Crosssectional or baseline assessment of longitudinal studies.

Eligibility criteria: Inclusion: 1. Studies with full text available in English language. 2. Cross-sectional study design or baseline data of longitudinal study design. 3 . Population: Subjects from any age group with permanent dentition. 4. FRP as reference (gold standard) compared to the PRP recordings for the same study subjects. 5. FRP had original data and assessed at 6 sites per tooth or at 4 interproximal sites of all teeth except third molar. 6. Assessment and reporting of any of the following outcomes: i. Prevalence of moderate and/or severe periodontitis calculated at the subject level or total subjects with disease and without disease. ii. Risk associations to periodontitis. iii. Mean and standard deviation for severity estimate. iv. Mean and standard deviation for extent estimate. 7. Use of Clinical Attachment Loss (CAL) to define the periodontal disease. Exclusion criteria: 1. Studies in foreign languages. 2. Simulation studies, hypothetical data or variables. 3. Subjects with primary dentition. 4. Measurements of periodontal disease are limited to periodontal pocket depth (PPD), Bleeding on Probing (BOP) or other periodontal parameters without assessment of CAL. 5. The reference FRP measured at less than 4 interproximal sites per tooth, or at subset of teeth rather than assessment of full mouth.

Information sources: Medline via OVID and PubMed, Embase through OVID, Grey literature at the Networked Digital Library of Theses and Dissertations, Proquest Dissertations and Theses Global, OpenGrey, Biosis, and Grey Matter. References of the individual articles and related (similar) articles.

Main outcome(s): 1. Accuracy of estimating prevalence of periodontitis at (CAL $3 \mathrm{~mm}+$ ) and/or (CAL $5 \mathrm{~mm}+$ ) assessed using sensitivity, specificity, positive predictive value (PPV), negative predictive value (NPV) and absolute bias. 2. Accuracy of estimating the risk associations using absolute bias and/or relative bias. 3 . Accuracy of estimating the extent of periodontitis (CAL $3 \mathrm{~mm}+$ ) and/or (CAL $5 \mathrm{~mm}+$ ) assessed using absolute bias. 4 . Accuracy of estimating the severity of periodontitis assessed using absolute bias. 
Quality assessment / Risk of bias analysis: Quality assessment of individual studies will be done using 23 items adopted from the Quality Assessment Tool for Diagnostic Accuracy Studies (QUADAS) used for diagnostic studies in systematic reviews and the Standards for Reporting of Diagnostic Accuracy (STARD) guidelines for diagnostic accuracy studies. In addition, few items were added specific to the use of PRP. Risk of bias of individual studies will be done using analytic approach.

Strategy of data synthesis: Narrative summary of the studies will be done including the study design, demographics, disparities between the studies' methodologies and implications of their findings. OpenMeta-Analyst software will be used for standard pairwise metaanalysis. Quantitative synthesis will be done for eligible studies to summarize the sensitivity and absolute bias of prevalence of periodontitis using different PRPs. The absolute bias of severity and extent estimates will be also quantified. A random effect model will be used due to the anticipated high level of heterogeneity in the studies' methodologies. Heterogeneity will be quantified using 12 and Chi-square p-value. Meta-bias will be assessed including the assessment of publication bias and selective reporting if applicable.

Subgroup analysis: A comparison based on the age, and certain subject characteristics will be done if applicable.

Sensitivity analysis: Periodontal disease defined at different threshold will be compared; Minimum number of sites used for defining the prevalence of periodontitis definition will also be compared.

Language: English.

Country(ies) involved: Canada and Saudi Arabia.

Keywords: Partial mouth recording; prevalence; risk associations; extent; severity.
Contributions of each author:

Author 1 - Yasmine Alawaji.

Email: alawajiya@student.ubc.ca

Author 2 - Abdulsalam Alshammari.

Email: shammariab@ksau-hs.edu.sa

Author 3 - Ricardo Carvalho.

Email: rickmc@dentistry.ubc.ca

Author 4 - Nesrine Mostafa.

Email: nmostafa@dentistry.ubc.ca

Author 5 - Jolanta Aleksejuniene.

Email: jolanta@dentistry.ubc.ca 